Internat. J. Math. \& Math. Sci.

Vol. 24, No. 1 (2000) 49-53

S0161171200003380

(C) Hindawi Publishing Corp.

\title{
ISHIKAWA ITERATION PROCESS WITH ERRORS FOR NONEXPANSIVE MAPPINGS IN UNIFORMLY CONVEX BANACH SPACES
}

\author{
DENG LEI and LI SHENGHONG
}

(Received 6 May 1999)

\begin{abstract}
We shall consider the behaviour of Ishikawa iteration with errors in a uniformly convex Banach space. Then we generalize the two theorems of Tan and Xu without the restrictions that $C$ is bounded and $\limsup _{n} s_{n}<1$.
\end{abstract}

Keywords and phrases. Uniformly convex Banach space, nonexpansive mapping, Ishikawa iteration process with errors.

2000 Mathematics Subject Classification. Primary 47H10; Secondary 40A05.

1. Introduction. Let $C$ be a closed convex subset of a Banach space $X$ and $T: C \rightarrow C$ be nonexpansive (that is, $\|T x-T y\| \leq\|x-y\|$ for all $x, y$ in $C$ ). In 1974, Ishikawa [1] introduced a new iteration process as

$$
x_{n+1}=t_{n} T\left(s_{n} T x_{n}+\left(1-s_{n}\right) x_{n}\right)+\left(1-t_{n}\right) x_{n}, \quad n=0,1,2, \ldots,
$$

where $\left\{t_{n}\right\}$ and $\left\{s_{n}\right\}$ are sequences in $[0,1]$ satisfying certain restrictions. The Mann iteration process is a special case of Ishikawa where $s_{n}=0$ for all $n \geq 0$ [4].

In 1993, Tan and Xu [7] obtained following result: let $C$ be a bounded closed convex subset of a uniformly convex Banach space $X, T: C \rightarrow C$ a nonexpansive mapping. If for any initial guess $x_{0}$ in $C,\left\{x_{n}\right\}$ defined by (1.1), with the restrictions that $\sum_{n=0}^{\infty} t_{n}\left(1-t_{n}\right)=\infty, \sum_{n=0}^{\infty} s_{n}\left(1-t_{n}\right)<\infty$, and $\limsup _{n} s_{n}<1$, then $\lim _{n \rightarrow \infty}\left\|x_{n}-T x_{n}\right\|$ $=0$.

Let $C$ be a closed convex subset of a Banach space $X$ and $T: C \rightarrow C$ be nonexpansive. For any given $x_{0} \in C$ the sequence $\left\{x_{n}\right\}$ defined by

$$
x_{n+1}=\alpha_{n} x_{n}+\beta_{n} T y_{n}+\gamma_{n} u_{n}, \quad y_{n}=\hat{\alpha}_{n} x_{n}+\hat{\beta}_{n} T x_{n}+\hat{\gamma}_{n} v_{n}, \quad n \geq 0 .
$$

is called the Ishikawa iteration sequence with errors. Here $\left\{u_{n}\right\}$ and $\left\{v_{n}\right\}$ are two bounded sequences in $C$, and $\left\{\alpha_{n}\right\},\left\{\beta_{n}\right\},\left\{\gamma_{n}\right\},\left\{\hat{\alpha}_{n}\right\},\left\{\hat{\beta}_{n}\right\}$, and $\left\{\hat{\gamma}_{n}\right\}$ are six sequences in $[0,1]$ satisfying the conditions

$$
\alpha_{n}+\beta_{n}+\gamma_{n}=\hat{\alpha}_{n}+\hat{\beta}_{n}+\hat{\gamma}_{n}=1 \text { for all } n \geq 0 .
$$

In particular, if $\hat{\beta}_{n}=\hat{\gamma}_{n}=0$ for all $n \geq 0$, the $\left\{x_{n}\right\}$ defined by

$$
x_{0} \in C, \quad x_{n+1}=\alpha_{n} x_{n}+\beta_{n} T x_{n}+\gamma_{n} u_{n}, \quad n \geq 0,
$$

is called the Mann iteration sequence with errors. 
REMARK 1.1. Note the Ishikawa and Mann iterative processes are all special cases of the Ishikawa and Mann iterative processes with errors.

It has been shown that if $C$ is a nonempty bounded closed convex subset of a uniformly convex Banach space $X$, then every nonexpansive mapping $T: C \rightarrow C$ has a fixed point (see [2]). In this paper, we first extend [7, Lemma 2.3] to the Ishikawa iteration sequence with errors (1.2), without the restrictions that $C$ is bounded and $\limsup _{n} s_{n}<1$. Then we generalize [7, Theorems 3.1, 3.2, and 3.4].

\section{Lemmas}

LEMMA 2.1. Suppose that $\left\{a_{n}\right\},\left\{b_{n}\right\}$, and $\left\{c_{n}\right\}$ are three sequences of nonnegative numbers such that

$$
a_{n+1} \leq\left(1+b_{n}\right) a_{n}+c_{n} \text { for all } n \geq 1 \text {. }
$$

If $\sum_{n=1}^{\infty} b_{n}$ and $\sum_{n=1}^{\infty} c_{n}$ converges, then $\lim _{n \rightarrow \infty} a_{n}$ exists.

Proof. For $n, m \geq 1$, we have

$$
\begin{aligned}
a_{n+m+1} & \leq\left(1+b_{n+m}\right) a_{n+m}+c_{n+m} \\
& \leq \prod_{i=n}^{n+m}\left(1+b_{i}\right) a_{n}+\sum_{i=n}^{n+m} \prod_{j=i+1}^{n+m}\left(1+b_{j}\right) c_{i} \\
& \leq \cdots \leq \prod_{i=n}^{n+m}\left(1+b_{i}\right) a_{n}+\prod_{j=n}^{n+m}\left(1+b_{j}\right) \sum_{i=n}^{n+m} c_{i} .
\end{aligned}
$$

It follows that

$$
\limsup _{m \rightarrow \infty} a_{m} \leq \prod_{i=n}^{\infty}\left(1+b_{i}\right) a_{n}+\prod_{j=n}^{\infty}\left(1+b_{j}\right) \sum_{i=n}^{\infty} c_{i} .
$$

Hence, $\limsup _{m \rightarrow \infty} a_{m} \leq \liminf _{n \rightarrow \infty} a_{n}$. This completes the proof.

LEMMA 2.2. Let $C$ be a closed convex subset of a Banach space $X$, and let $T: C \rightarrow X$ a nonexpansive mapping. Then for any initial guess $x_{0}$ in $C,\left\{x_{n}\right\}$ defined by (1.2),

$$
\left\|x_{n+1}-p\right\| \leq\left\|x_{n}-p\right\|+\gamma_{n}\left\|u_{n}-p\right\|+\beta_{n} \hat{\gamma}_{n}\left\|v_{n}-p\right\|,
$$

for all $n \geq 1$ and for all $p \in F(T)$, where $F(T)$, denotes the set of fixed points of $T$.

Proof. For all $p \in F(T)$, we have

$$
\begin{aligned}
& \left\|x_{n+1}-p\right\| \\
& \quad \leq \alpha_{n}\left\|x_{n}-p\right\|+\beta_{n}\left\|T y_{n}-p\right\|+\gamma_{n}\left\|u_{n}-p\right\| \\
& \quad \leq \alpha_{n}\left\|x_{n}-p\right\|+\beta_{n}\left(\hat{\alpha}_{n}\left\|x_{n}-p\right\|+\hat{\beta}_{n}\left\|T x_{n}-p\right\|+\hat{\gamma}_{n}\left\|v_{n}-p\right\|\right)+\gamma_{n}\left\|u_{n}-p\right\| \\
& \quad \leq\left\|x_{n}-p\right\|+\gamma_{n}\left\|u_{n}-p\right\|+\beta_{n} \hat{\gamma}_{n}\left\|v_{n}-p\right\| .
\end{aligned}
$$

This completes the proof.

LEMMA 2.3 [3]. Let $C$ be a closed convex subset of a uniformly convex Banach space $X$, and let $T: C \rightarrow X$ a nonexpansive mapping. Then the mapping $I-T$ is demiclosed on $C$. 


\section{Main Results}

THEOREM 3.1. Let $C$ be a closed convex subset of a uniformly convex Banach space $X, T: C \rightarrow C$ a nonexpansive mapping with a fixed point. If for any initial guess $x_{0}$ in $C,\left\{x_{n}\right\}$ defined by (1.2), with the restrictions that $\sum_{n=0}^{\infty} \alpha_{n} \beta_{n}=\infty, \sum_{n=0}^{\infty} \alpha_{n} \hat{\beta}_{n}<$ $\infty, \sum_{n=0}^{\infty} \gamma_{n}<\infty$ and $\sum_{n=0}^{\infty} \hat{\gamma}_{n}<\infty$, then $\lim _{n \rightarrow \infty}\left\|x_{n}-T x_{n}\right\|=0$.

Proof. By Lemma 2.2 and $T$ with a fixed point, we set

$$
M=\sup _{n \geq 0}\left(\left\|T x_{n}-u_{n}\right\|,\left\|x_{n}-u_{n}\right\|,\left\|T y_{n}-v_{n}\right\|,\left\|y_{n}-u_{n}\right\|,\left\|x_{n}-v_{n}\right\|\right)
$$

It follows from (1.2) that

$$
\begin{aligned}
\left\|x_{n+1}-T x_{n+1}\right\| \leq & \alpha_{n}\left\|x_{n}-T x_{n+1}\right\|+\beta_{n}\left\|T y_{n}-T x_{n+1}\right\|+\gamma_{n}\left\|T x_{n+1}-u_{n}\right\| \\
\leq & \alpha_{n}\left(\left\|x_{n}-T x_{n}\right\|+\left\|T x_{n}-T x_{n+1}\right\|\right) \\
& +\beta_{n}\left(\alpha_{n}\left\|y_{n}-x_{n}\right\|+\beta_{n}\left\|y_{n}-T y_{n}\right\|+\gamma_{n}\left\|y_{n}-u_{n}\right\|\right)+\gamma_{n} M \\
\leq & \alpha_{n}\left(\left\|x_{n}-T x_{n}\right\|+\beta_{n}\left\|x_{n}-T y_{n}\right\|\right)+\alpha_{n} \beta_{n} \hat{\beta}_{n}\left\|x_{n}-T x_{n}\right\| \\
& +\beta_{n}^{2}\left(\hat{\alpha}_{n}\left\|x_{n}-T y_{n}\right\|+\hat{\beta}_{n}\left\|T x_{n}-T y_{n}\right\|\right)+\gamma_{n} M+\beta_{n} \gamma_{n}\left\|y_{n}-u_{n}\right\| \\
& +\alpha_{n} \gamma_{n}\left\|x_{n}-u_{n}\right\|+\alpha_{n} \beta_{n} \hat{\gamma}_{n}\left\|x_{n}-v_{n}\right\|+\beta_{n}^{2} \hat{\gamma}_{n}\left\|T y_{n}-v_{n}\right\| \\
\leq & \alpha_{n}\left\|x_{n}-T x_{n}\right\|+\alpha_{n} \beta_{n}\left\|x_{n}-T y_{n}\right\|+\alpha_{n} \beta_{n} \hat{\beta}_{n}\left\|x_{n}-T x_{n}\right\| \\
& +\beta_{n}^{2} \hat{\alpha}_{n}\left\|x_{n}-T y_{n}\right\|+\beta_{n}^{2} \hat{\beta}_{n}\left\|x_{n}-y_{n}\right\|+2 \gamma_{n} M+\beta_{n} \hat{\gamma}_{n} M \\
\leq & \left(\alpha_{n}+\alpha_{n} \beta_{n} \hat{\beta}_{n}+\beta_{n}^{2} \hat{\beta}_{n}^{2}\right)\left\|x_{n}-T x_{n}\right\| \\
& +\left(\alpha_{n} \beta_{n}+\beta_{n}^{2} \hat{\alpha}_{n}\right)\left(\left\|x_{n}-T x_{n}\right\|+\left\|T x_{n}-T y_{n}\right\|\right) \\
& +2 \gamma_{n} M+\beta_{n} \hat{\gamma}_{n} M+\beta_{n}^{2} \hat{\beta}_{n} \hat{\gamma}_{n} M \\
\leq & \left(\alpha_{n}+\alpha_{n} \beta_{n} \hat{\beta}_{n}+\beta_{n}^{2} \hat{\beta}_{n}^{2}+\alpha_{n} \beta_{n}+\beta_{n}^{2} \hat{\alpha}_{n}+\alpha_{n} \beta_{n} \hat{\beta}_{n}+\beta_{n}^{2} \hat{\alpha}_{n} \hat{\beta}_{n}\right) \\
& \times\left\|x_{n}-T x_{n}\right\|+2 \gamma_{n} M+\beta_{n} \hat{\gamma}_{n} M+\left(\alpha_{n} \beta_{n}+\beta_{n}^{2} \hat{\alpha}_{n}+\beta_{n}^{2} \hat{\beta}_{n}\right) \hat{\gamma}_{n} M \\
\leq & \left(1+2 \alpha_{n} \beta_{n} \hat{\beta}_{n}\right)\left\|x_{n}-T x_{n}\right\|+2\left(\gamma_{n}+\beta_{n} \hat{\gamma}_{n}\right) M .
\end{aligned}
$$

Setting $a_{n}=T x_{n}-x_{n}, b_{n}=2 \alpha_{n} \beta_{n} \hat{\beta}_{n}$, and $c_{n}=2\left(\gamma_{n}+\beta_{n} \hat{\gamma}_{n}\right) M$, it follows from Lemma 2.1 that $\lim _{n \rightarrow \infty}\left\|a_{n}\right\|$ exists.

Let $r\left(x_{0}\right)=\lim _{n \rightarrow \infty}\left\|x_{n}-T x_{n}\right\|$. To reach the desired conclusion, it suffices to show that $r\left(x_{0}\right)$ is independent of the initial value $x_{0}$. We let $\left\{x_{n}^{*}\right\}$ denote iteration (1.2) commencing at $x_{0}^{*}$. Since $\left\|x_{n+1}-x_{n+1}^{*}\right\| \leq\left\|x_{n}-x_{n}^{*}\right\|$, we may assume that $\lim _{n \rightarrow \infty} \| x_{n}-$ $x_{n}^{*} \|=d>0$. Then, we obtain

$$
\begin{aligned}
\left\|x_{n+1}-x_{n+1}^{*}\right\| & =\left\|\alpha_{n}\left(x_{n}-x_{n}^{*}\right)+\beta_{n}\left(T y_{n}-T y_{n}^{*}\right)\right\| \\
& \leq\left[1-2 \alpha_{n} \beta_{n} \delta\left(\frac{\left\|x_{n}-x_{n}^{*}-\left(T y_{n}-T y_{n}^{*}\right)\right\|}{\left\|x_{n}-x_{n}^{*}\right\|}\right)\right]\left\|x_{n}-x_{n}^{*}\right\|,
\end{aligned}
$$

since $\left\|T y_{n}-T y_{n}^{*}\right\| \leq\left\|x_{n}-x_{n}^{*}\right\|$. Thus,

$$
\sum_{i=0}^{n} 2 \alpha_{i} \beta_{i} \delta\left(\frac{\left\|x_{i}-x_{i}^{*}-\left(T y_{i}-T y_{i}^{*}\right)\right\|}{\left\|x_{i}-x_{i}^{*}\right\|}\right)\left\|x_{i}-x_{i}^{*}\right\| \leq\left\|x_{0}-x_{0}^{*}\right\|-\left\|x_{n+1}-x_{n+1}^{*}\right\| .
$$


It follows that

$$
\sum_{n=0}^{\infty} \alpha_{n} \beta_{n} \delta\left(\frac{\left\|x_{n}-x_{n}^{*}-\left(T y_{n}-T y_{n}^{*}\right)\right\|}{\left\|x_{n}-x_{n}^{*}\right\|}\right)<\infty .
$$

By condition $\sum_{n=0}^{\infty} \alpha_{n} \hat{\beta_{n}}<\infty$, we have $\sum_{n=0}^{\infty} \alpha_{n} \beta_{n} \hat{\beta}_{n}<\infty$. Thus,

$$
\sum_{n=0}^{\infty} \alpha_{n} \beta_{n}\left\{\delta\left[\frac{\left\|x_{n}-x_{n}^{*}-\left(T y_{n}-T y_{n}^{*}\right)\right\|}{\left\|x_{n}-x_{n}^{*}\right\|}\right]+\hat{\beta}_{n}\right\}<\infty .
$$

It follows that

$$
\liminf _{n \rightarrow 0}\left[\left\|x_{n}-x_{n}^{*}-\left(T y_{n}-T y_{n}^{*}\right)\right\|+\hat{\beta}_{n}\right]=0
$$

since $\sum_{n=0}^{\infty} \alpha_{n} \beta_{n}=\infty$ and $\delta$ is the modulus of convexity of uniformly convex Banach space $X$. Hence, there is a sequence $\left\{n_{k}\right\} \subset\{n\}$ such that

$$
\lim _{k \rightarrow \infty}\left\|x_{n_{k}}-x_{n_{k}}^{*}-\left(T y_{n_{k}}-T y_{n_{k}}^{*}\right)\right\|=0, \quad \lim _{k \rightarrow \infty} \hat{\beta}_{n_{k}}=0 .
$$

On the other hand, we have

$$
\begin{aligned}
\| x_{n_{k}} & -T x_{n_{k}}\|-\| x_{n_{k}}^{*}-T x_{n_{k}}^{*} \| \mid \\
& \leq\left\|\left(x_{n_{k}}-T x_{n_{k}}\right)-\left(x_{n_{k}}^{*}-T x_{n_{k}}^{*}\right)\right\| \\
& \leq\left\|x_{n_{k}}-x_{n_{k}}^{*}-\left(T y_{n_{k}}-T y_{n_{k}}^{*}\right)\right\|+\left\|T x_{n_{k}}-T y_{n_{k}}\right\|+\left\|T x_{n_{k}}^{*}-T y_{n_{k}}^{*}\right\| \\
& \leq\left\|x_{n_{k}}-x_{n_{k}}^{*}-\left(T y_{n_{k}}-T y_{n_{k}}^{*}\right)\right\|+\hat{\beta}_{n_{k}}\left\|x_{n_{k}}-T x_{n_{k}}\right\|+\hat{\beta}_{n_{k}}\left\|x_{n_{k}}^{*}-T x_{n_{k}}^{*}\right\|+2 \hat{\gamma}_{n} M .
\end{aligned}
$$

Setting $k \rightarrow \infty$ in (3.9), it follows from (3.8) that

$$
\lim _{k \rightarrow \infty}||\left|x_{n_{k}}-T x_{n_{k}}\|-\| x_{n_{k}}^{*}-T x_{n_{k}}^{*}\right|||=0 .
$$

Thus,

$$
\lim _{n \rightarrow \infty}\left|\left\|x_{n}-T x_{n}\right\|-\left\|x_{n}^{*}-T x_{n}^{*}\right\|\right|=0,
$$

that is, $r\left(x_{0}\right)=r\left(x_{0}^{*}\right)$. This completes the proof.

Recall that a Banach space $X$ is said to satisfy Opial's condition [5] if the condition $x_{n} \rightarrow x_{0}$ weakly implies

$$
\limsup _{n \rightarrow \infty}\left\|x_{n}-x_{0}\right\|<\limsup _{n \rightarrow \infty}\left\|x_{n}-y\right\| \text { for all } y \neq x_{0} .
$$

THEOREM 3.2. Let $C$ be a closed convex subset of a uniformly convex Banach space $X$ which satisfies Opial's condition, $T: C \rightarrow C$ a nonexpansive mapping with a fixed point, and $\left\{x_{n}\right\}$ as in Theorem 3.1. Then $\left\{x_{n}\right\}$ converges weakly to a fixed point of $T$.

Proof. Let $\omega_{w}\left(x_{n}\right)$ be the weak limit $\omega$-set of $\left\{x_{n}\right\}$. By Lemma 2.3 and Theorem 3.1, $\omega_{w}\left(x_{n}\right)$ is contained in $F(T)$, the fixed point set of $T$.

The remainder of the proof is similar to that of [7, Theorem 3.1], so the details are omitted. 
REMARK 3.3. Theorem 3.2 generalizes [7, Theorem 3.1].

Recall that a mapping $T: C \rightarrow C$ with a nonempty fixed points set $F(T)$ in $C$ will be said to satisfy condition $A[6]$ if there is a nondecreasing function $f:[0, \infty) \rightarrow[0, \infty)$ with $f(0)=0, f(r)>0$ for $r \in(0, \infty)$, such that $\|x-T x\| \geq f(d(x, F(T)))$ for all $x \in C$, where $d(x, F(T))=\inf \{\|x-z\|: z \in F(T)\}$.

The following two theorems generalize Theorem 3.2 and [7, Theorem 3.4] respectively. Since a similar proof is in [7], we omit their proof here.

THEOREM 3.4. Let $X, C, T$, and $\left\{x_{n}\right\}$ be as in Theorem 3.1. If the range of $C$ under $T$ is contained in a compact subset of $X$, then $\left\{x_{n}\right\}$ converges strongly to a fixed point of $T$.

THEOREM 3.5. Let $X, C, T$ and $\left\{x_{n}\right\}$ be as in Theorem 3.1. If $T$ with a nonempty fixed points set $F(T)$ satisfies condition $A$, then $\left\{x_{n}\right\}$ converges strongly to a fixed point of $T$.

ACKNOWLEDGEMENT. Research partially supported by NNSF(79790130) and ZJPNSF(198013).

\section{REFERENCES}

[1] S. Ishikawa, Fixed points by a new iteration method, Proc. Amer. Math. Soc. 44 (1974), 147-150. MR 49\#1243. Zbl 286.47036.

[2] W. A. Kirk, A fixed point theorem for mappings which do not increase distances, Amer. Math. Monthly 72 (1965), 1004-1006. MR 32\#6436. Zbl 141.32402.

[3] _ Nonexpansive mappings in product spaces, set-valued mappings and $k$-uniform rotundity, Nonlinear Functional Analysis and its Applications, Part 2 (Berkeley, Calif., 1983) (Providence, R.I.), Amer. Math. Soc., 1986, pp. 51-64. MR 87i:47068. Zbl 594.47048.

[4] W. R. Mann, Mean value methods in iteration, Proc. Amer. Math. Soc. 4 (1953), 506-510. MR 14,988f. Zbl 050.11603.

[5] Z. Opial, Weak convergence of the sequence of successive approximations for nonexpansive mappings, Bull. Amer. Math. Soc. 73 (1967), 591-597. MR 35\#2183. Zbl 179.19902.

[6] H. F. Senter and W. G. Dotson, Jr., Approximating fixed points of nonexpansive mappings, Proc. Amer. Math. Soc. 44 (1974), 375-380. MR 49\#11333. Zbl 299.47032.

[7] K. K. Tan and H. K. Xu, Approximating fixed points of nonexpansive mappings by the Ishikawa iteration process, J. Math. Anal. Appl. 178 (1993), no. 2, 301-308. MR 94g:47076. Zbl 895.47048.

Deng Lei: Department of Mathematics, Southwest China Normal University, Beibei, CHONGQING 400715, CHINA

Li SHENGHONG: DEPARTMENT OF MATHEMATICS, ZheJIANG UNIVERSITY HANGZHOU, 310027, CHINA

E-mail address: 1sh@math.zju. edu.cn 


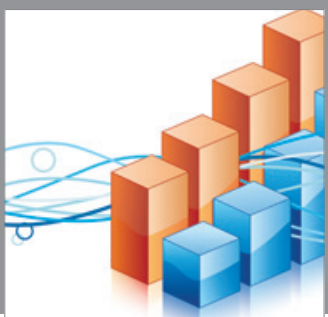

Advances in

Operations Research

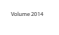

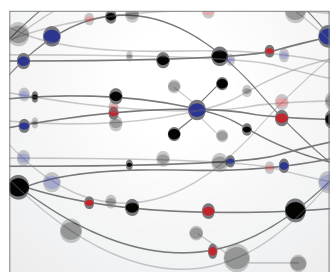

\section{The Scientific} World Journal
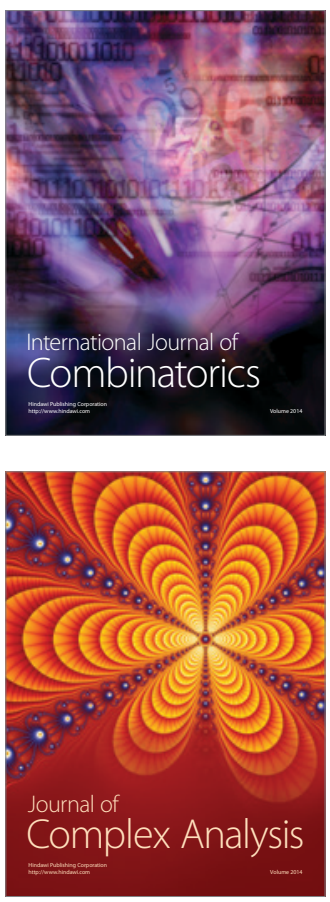

International Journal of

Mathematics and

Mathematical

Sciences
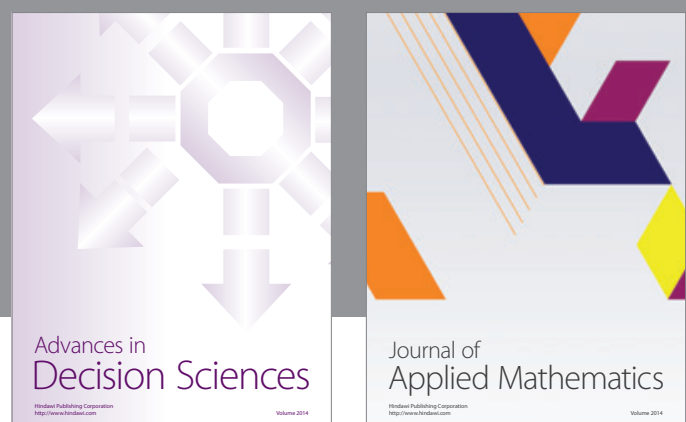

Journal of

Applied Mathematics
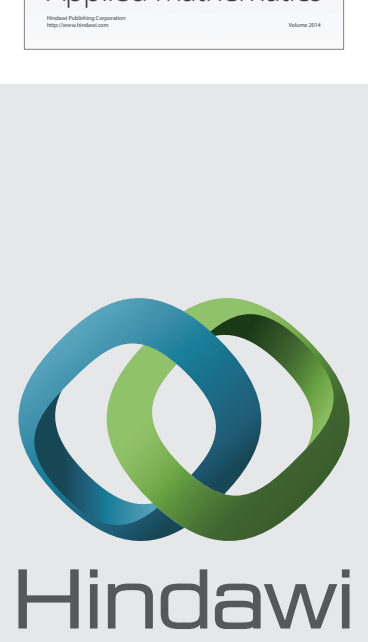

Submit your manuscripts at http://www.hindawi.com
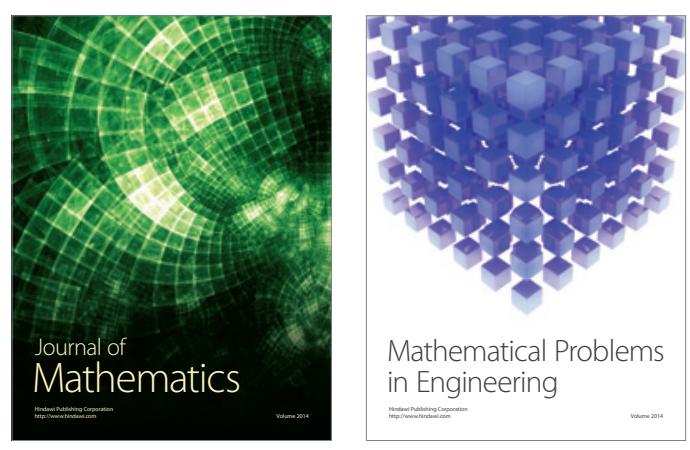

Mathematical Problems in Engineering
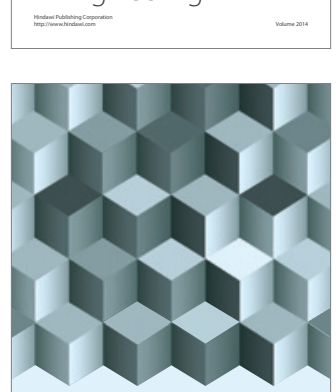

Journal of

Function Spaces
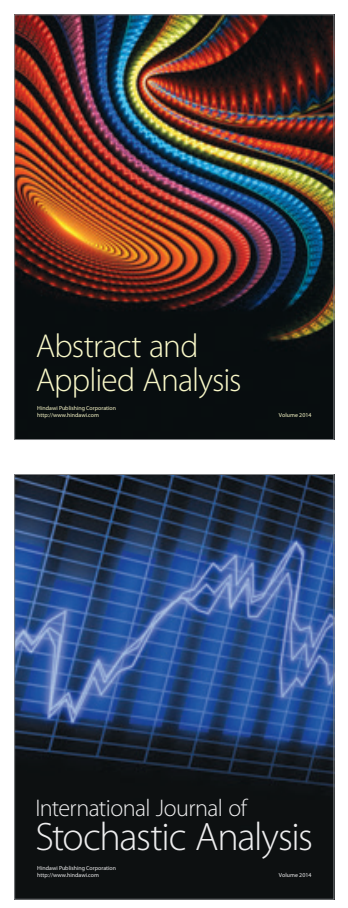

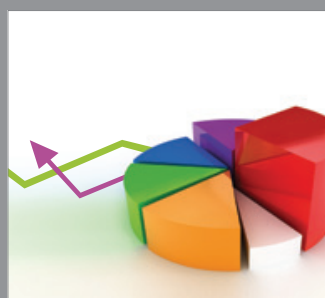

ournal of

Probability and Statistics

Promensencen
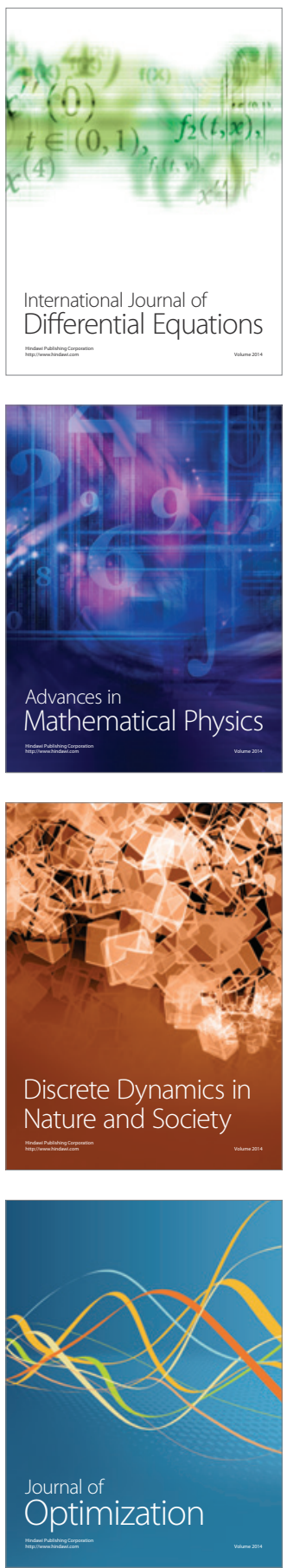